\title{
Editorial
}

\section{Editorial to Physiotherapy Practice and Research volume 42 issue 2}

Thank you for taking the time to read the latest issue of Physiotherapy Practice and Research. As we come close to the holidays and the New Year, we would like to wish you every bit of luck in the subsequent year.

We look forward to you taking the time to read this volume. Firstly, we have a systematic review and meta-analysis on exercise as a treatment for clinical depression, this article also makes a convincing argument for the progression of the physiotherapists role into mental health and aiding with treating depression. Following this we have two randomized controlled trials looking at glenohumeral and scapulothoracic mobilisation with stretching in overhead throwing athletes with glenohumeral internal rotation deficit, and early rehabilitation. Next up, we have a review of current prehabilitation guidelines for patients awaiting elective total knee replacement in the United Kingdom's National Health Service. Physiotherapists' knowledge of current sport related concussion guidelines and management was assessed and reported that many physiotherapists worldwide are aware of these guidelines.
For those interested in managing falls, we have an interesting mixed-methods study on older adult's experience using active computer games to prevent falls. There is also an exploratory study on the association between recently diagnosed cancer and incidence of falling in older adults.

To round this volume, we have articles on airway clearance techniques for acute exacerbation of chronic obstructive pulmonary disease, nurses and physiotherapy assistants attitudes' toward low back pain treatment, and a feasibility study on an education session to manage patellofemoral pain.

Thank you for reading Physiotherapy Practice and Research, we hope you enjoy this issue and future issues to come.

Dr Orlagh O'Shea

Dr Jonathan Moran Editors-in-Chief 